\title{
The Study to Investigate Growth Rate of Metal Nanowires into Anodic Alumina Oxide Arrays via Electrodeposition
}

\author{
Tahir Mehmood, Aiman Mukhtar, Babar Khan, Kaiming Wu \\ The State Key Laboratory of Refractories and Metallurgy Hubei Province, Key Laboratory of Systems Science in Metallurgical Process, \\ International Research Institute for Steel Technology, Wuhan University of Science and Technology, Wuhan, P. R. China
}

\section{Email address:}

wukaiming@wust.edu.cn (Kaiming Wu)

${ }^{*}$ Corresponding author

\section{To cite this article:}

Tahir Mehmood, Aiman Mukhtar, Babar Khan, Kaiming Wu. The Study to Investigate Growth Rate of Metal Nanowires into Anodic Alumina Oxide Arrays via Electrodeposition. American Journal of Chemical Engineering. Vol. 4, No. 2, 2016, pp. 57-61. doi: 10.11648/j.ajche.20160402.15

Received: May 6, 2016; Accepted: May 17, 2016; Published: May 19, 2016

\begin{abstract}
To understand the growth mechanism of electrodeposited $\mathrm{Fe}, \mathrm{Ni}$ and $\mathrm{Co}$, we have studied the effect of workfunction on the metal nanowire growth by SEM and Potentiostat. We found that the current density for deposition of Fe nanowires is higher than of $\mathrm{Ni}$ and $\mathrm{Co}$ nanowires under the same fixed potential, overpotential, $\mathrm{pH}$, concentration of metal ions and temperature. Using the electrons tunneling theory, we have argued that the current density of the metals depends on the workfunction and width of double layer. The current density increases with decreasing the length of double layer. The current density of metal with a smaller workfunction can be higher than that of metal with a larger workfunction.
\end{abstract}

Keywords: Nanostructure, Electrodeposition, Current Density, Work Function

\section{Introduction}

Metal nanowires have attracted a great deal of research interest in recent years because of their electronic and optical properties [1] and potential applications in nanodevices [2]. Several groups prepared and studied the silver nanowires or nanorods using templates [1-3]. Currently, the synthesis of nanowires by the electrochemical deposition is of great interest. To fabricate and investigate the growth mechanisms of metal nanowires in the cylindrical pores of a template is of essential and technological interests [4]. Template commonly used for nanowire synthesis is Anodic Aluminum Oxide (AAO) owing to its salient features such as decorative properties, nanopore symmetry and corrosion resistance $[5,6]$. In comparison to some other deposition procedures, AAO template method is more inexpensive and allows to generate very thin nanowires [7] Controlling the growth of metal nanowires is important to achieve these applications. Therefore, understanding various factors that influence the growth of electrodeposited metal nanowires is of scientific and technological interests. Deposition potential plays an important role in controlling the metal nanowire growth. For example, the nanowires of $\mathrm{Au}, \mathrm{Ag}$, and $\mathrm{Cu}$ are single crystalline under the low deposition potential, and polycrystalline under the high deposition potential [8]. We have proposed in the previous work that the growth of metal nanowires can be pictured as the four steps at the atomic-level scale [9]. The dehydration of hydrated metal ions can be the most important among the four steps. The dehydration involves valence electrons tunneling to hydrated metal ions [9, 10], leading to neutralization of the hydrated metal ions. The neutral metal atoms are adsorbed on the surface and then diffuse to surface sites (such as kink site) where they incorporate into the metal lattice, thus leading to the growth [9]. Therefore, the growth rate is related by the dehydration [9]. Since the probability of electron tunneling is related to the workfunction of metals, the workfunction can have a significant effect on the growth of metal nanowires. This paper offers different parameters that may be responsible for the fast growth of metal nanowires.

\section{Experimental Details}

The porous anodic alumina membranes (AAM) templates were prepared using a two-step anodization procedure $[11,12]$ as shown in Fig. 1. 
A I substrate

a)

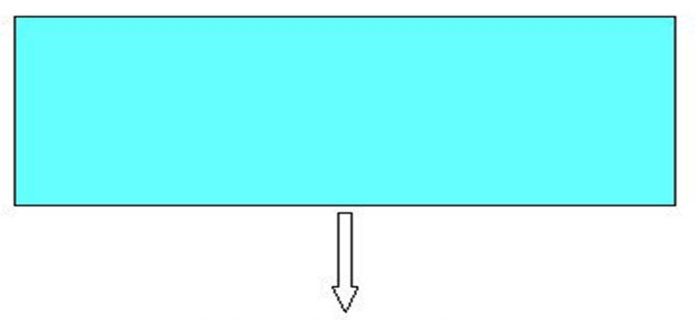

1 st Anodization

b)

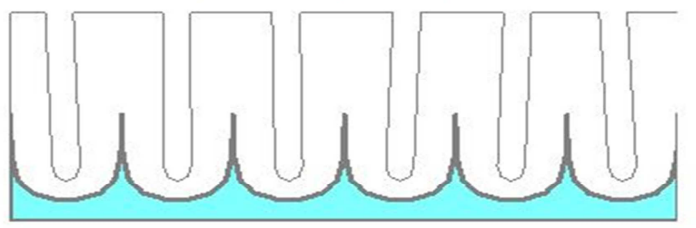

$\sqrt{\zeta}$

Etching of $A A O$

c)

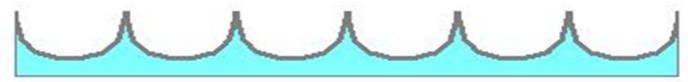

2 nd Anodization

d)

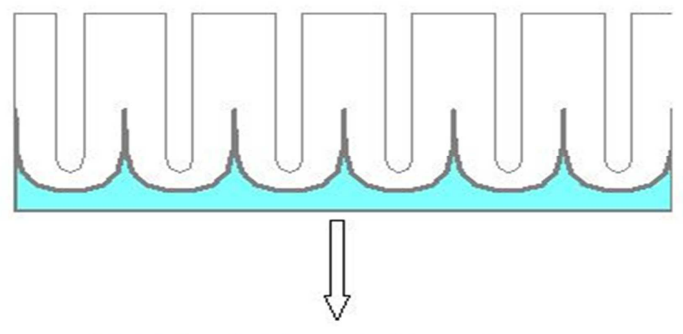

Thinning of $A A O$ barrier

e)

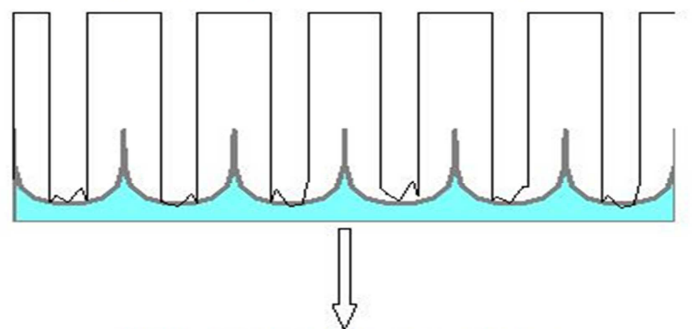

NW Electrodeposition

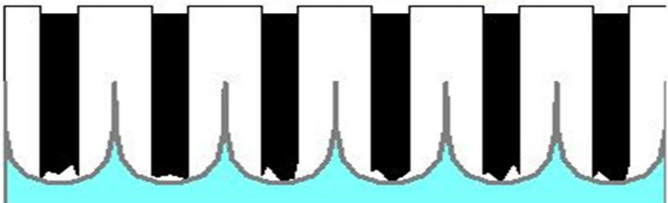

Fig. 1. Schematic view of the process flow used for AAO template formation (a) Al foil (b) first anodization step (c) removal of first AAO layer, (d) second anodization step and AAO membranes; before gold sputtering (e), and after gold sputtering (f).

Firstly, high-purity aluminum foils (99.999\%) were degreased in acetone and then were annealed and secondly, the aluminum foils were anodized two times. Thirdly, the alumina barrier layer was then dissolved in a $5 \mathrm{wt} \%$ phosphoric acid solution at $40^{\circ} \mathrm{C}$. The templates obtained by this procedure have the cylindrical and hexagonally arranged pores. The average diameter and spacing of pores are estimated to be 50 $\mathrm{nm}$ and $100 \mathrm{~nm}$. Assuming that the pore distribution is exactly hexagonal, the ratio of the area of a pore ( $\mathrm{S}_{\text {pore }}=\pi(25 \mathrm{~nm})^{2}$ where $25 \mathrm{~nm}$ is the pore radius) to the template area that this pore possesses $\left(\mathrm{S}_{\text {template }}=100 \mathrm{~nm} \cdot 100 \mathrm{~nm} \cdot \sin 60^{\circ}\right.$ where $100 \mathrm{~nm}$ is the distance between two pores), $\mathrm{S}_{\text {pore }} / \mathrm{S}_{\text {template }}$, was calculated to be 0.225 . The length of the pore is about $65 \mu \mathrm{m}$. The length of nanowires was estimated to be about $60 \mu \mathrm{m}$. A gold (Au) film was sputtered onto the back side of the templates to serve as the working electrode [13]. Finally, a gold film was sputtered onto the back side of the templates to serve as the working electrode.

The electrolytes have the same concentration of metal ions. The electrolyte was $0.536 \mathrm{M} \quad \mathrm{FeSO}_{4} \cdot 7 \mathrm{H}_{2} \mathrm{O}$ and $0.72 \mathrm{M}$ $\mathrm{H}_{3} \mathrm{BO}_{3}$ for deposition of $\mathrm{Fe}$ nanowires, $0.536 \mathrm{M}$ $\mathrm{CoSO}_{4} \cdot 7 \mathrm{H}_{2} \mathrm{O}$ and $0.72 \mathrm{M} \mathrm{H} \mathrm{H}_{3} \mathrm{BO}_{3}$ for deposition of $\mathrm{Co}$ nanowires and $0.536 \mathrm{M} \mathrm{NiSO} \cdot 6 \mathrm{H}_{2} \mathrm{O}$ and $0.72 \mathrm{M} \mathrm{H}_{3} \mathrm{BO}_{3}$ for deposition of $\mathrm{Ni}$ nanowires. The $\mathrm{pH}$ of the two as-prepared solutions was adjusted to 2 by adding $1 \mathrm{M} \mathrm{H}_{2} \mathrm{SO}_{4}$ solution. Direct current electrodeposition (shown in Fig. 2) was conducted in a three-electrode cell at room temperature.

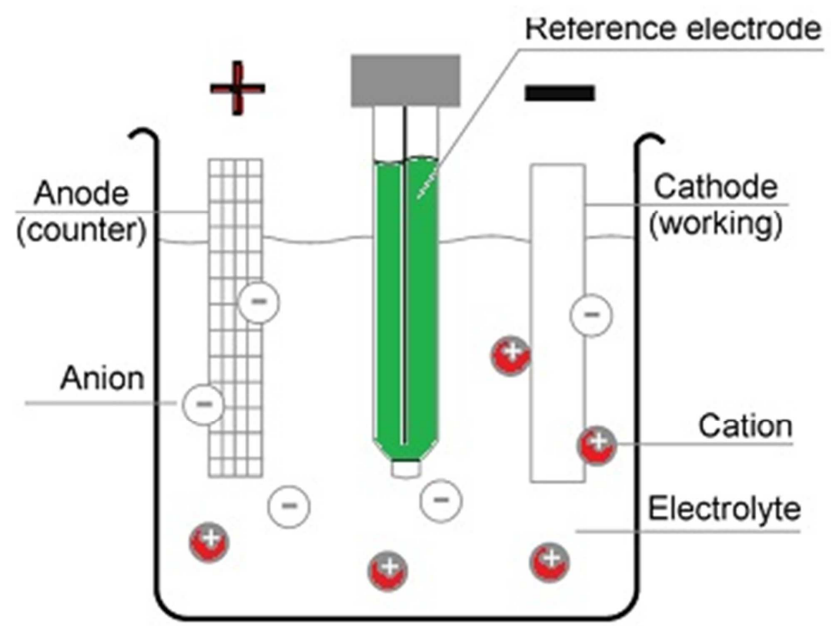

Fig. 2. Schematic representation of a three electrode electrochemical cell.

The area of the working electrode for growth of nanowires was $0.608 \mathrm{~cm}^{2}\left(=0.25 \pi(0.88 \mathrm{~cm})^{2}\right)$ and the area of the graphite counter electrode was $14.7 \mathrm{~cm}^{2}(=4.2 \mathrm{~cm} \times 3.5 \mathrm{~cm})$. The reference electrode was the saturated calomel. The deposited $\mathrm{Fe}$, $\mathrm{Co}$ and $\mathrm{Ni}$ nanowires were also examined by scanning electron microscope (SEM, JEOL JSM-6700F). In order to perform SEM observations, the AAO template was partly dissolved with a $5 \mathrm{wt} \% \mathrm{NaOH}$ solution, and then carefully rinsed with deionized water for several times. 


\section{Results and Discussions}

In the electrodeposition of metal, a metal ion $\mathrm{Mn}+$ is transferred from solution into the ionic metal lattice, meanwhile electrons are provided from the external electron source (power supply) to the electron gas of the metal M [14]. This electrodeposition process can be pictured as the following four steps in the atomic-level scale. First, hydrated metal ions in an aqueous solution diffuse to a metal surface and are adsorbed on this surface. (In hydrated metal ions, the water molecules of hydration are electrostatically attached to the metal ions.) Second, when an adsorbed hydrated metal ion captures electrons from the surface by quantum-mechanical tunneling, the metal ion becomes a neutral metal atom. The electrostatic attractive interaction between the neutral metal atom and water molecules is zero and the water molecules of hydration are displaced. Third, the neutral metal atom adsorbed on the surface. Fourth, an adsorbed metal atom diffuses to a surface site (such as kink site) where it incorporates into the ionic metal lattice, leading to the growth of nanowires. The current density arises from valence electron tunneling from the metal surface to hydrated metal and hydrogen ions, leading metal nanowire growth and hydrogen evolution, respectively.

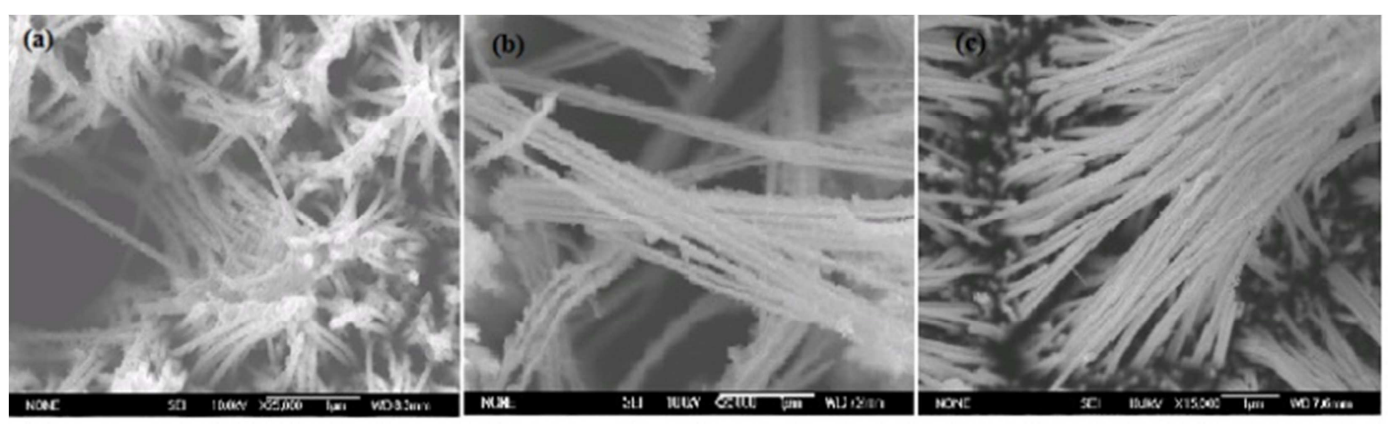

Fig. 3. SEM images of $\mathrm{Fe}(\mathrm{a}) \mathrm{Ni}(\mathrm{b})$ and $\mathrm{Co}(\mathrm{c})$ nanowires deposited at $-0.6 \mathrm{~V}$.

Fig. 3 shows the SEM images of $\mathrm{Fe}, \mathrm{Ni}$ and Co nanowires. The diameter of Fe nanowires $(\sim 50 \mathrm{~nm})$ is the same as that of the pores of AAO template $(\sim 50 \mathrm{~nm})$, indicating that the cylindrical pores of the AAO template were fully filled with Fe metal atoms during electrodeposition.

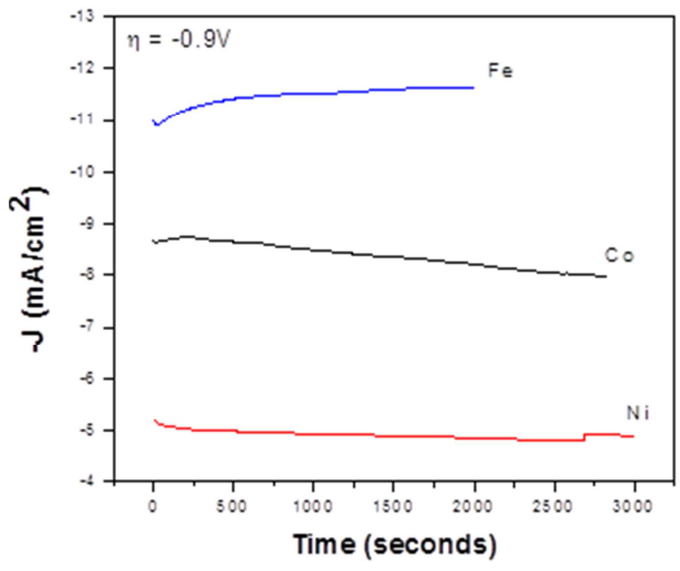

Fig. $4(a, b)$ shows the current density against time curves measured in the second-step deposition of $\mathrm{Fe}, \mathrm{Co}$ and $\mathrm{Ni}$ nanowires at the overpotential $(\eta)$ of $-0.9 \mathrm{~V}$ and $-0.6 \mathrm{~V}$ respectively.

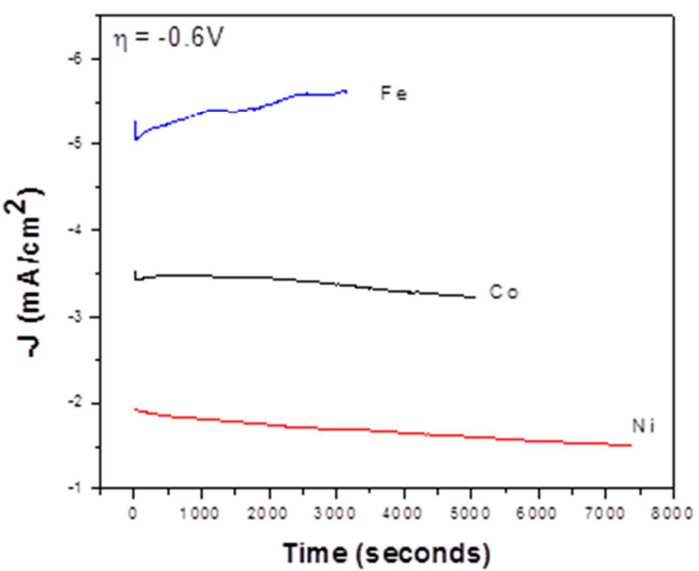

Fig. 4. (a, b) Current density against time curves measured in the second-step deposition of Fe, Co and Ni nanowires at $-0.9 \mathrm{~V}$ and at $-0.6 \mathrm{~V}$.

The averaged current density at $-0.9 \mathrm{~V}$ is $11.47 \mathrm{~mA} / \mathrm{cm}^{2}$ for $\mathrm{Fe}, 8.34 \mathrm{~mA} / \mathrm{cm}^{2}$ for $\mathrm{Co}, 4.92 \mathrm{~mA} / \mathrm{cm}^{2}$ for $\mathrm{Ni}$, and at $-0.6 \mathrm{~V}$ is $5.41 \mathrm{~mA} / \mathrm{cm}^{2}$ for $\mathrm{Fe}, 3.33 \mathrm{~mA} / \mathrm{cm}^{2}$ for Co and $1.61 \mathrm{~mA} / \mathrm{cm}^{2}$ for $\mathrm{Ni}$. We can see two interesting points from Figs. $4(\mathrm{a}, \mathrm{b})$ that under the same $\eta, \mathrm{pH}$, concentration of metal ions and temperature, the current density of deposition of Fe is higher than that of $\mathrm{Co}$ and $\mathrm{Ni}$, indicating that $\mathrm{Fe}$ nanowires grow faster than $\mathrm{Co}$ and $\mathrm{Ni}$ nanowires. In the following, we will explain this result.

The relationship between the current density and the overpotential is described by the Bulter-Volmer equation [15].
For the large negative values of overpotential like -0.6 and $-0.9 \mathrm{~V}$, the Bulter-Volmer equation can be approximated by

$$
\mathrm{i}=-\mathrm{i}_{0} \exp \left(-\frac{\alpha z \mathrm{~F} \eta}{\mathrm{RT}}\right)
$$

where $\mathrm{i}$ is the current density, $\mathrm{i}_{0}$ is the exchange current density, $\alpha$ is the charge transfer coefficient, $z$ is the valence of deposited metal ions, $F$ is the Faraday constant, $\eta$ is the over potential, $\mathrm{R}$ is the gas constant, and $\mathrm{T}$ is the absolute temperature. Exchange current density of $\mathrm{Ni}$ is approximately 
the same as that of Co at $\mathrm{pH} 2.5$ [16]. The valence of deposited $\mathrm{Ni}$ and Co ions, which is $2+$, is also the same. Consequently, the current density ( $i)$ depends only on the charge transfer coefficient $(\alpha)$ and increases with increasing $\alpha$. Here we will elucidate using the electron tunneling theory that the charge transfer coefficient of $\mathrm{Co}\left(\alpha_{\mathrm{Co}}\right)$ is higher than that of $\mathrm{Ni}$ $\left(\alpha_{\mathrm{Ni}}\right)$.

Electron tunneling is used to explain electronic processes occurring near metal surface. When positively charged ions approach a metal surface, metal valence electrons can neutralize these ions via electron tunneling. For example, in low-energy ion scattering experiments, low energy ions that are incident on a metal surface are neutralized via electron tunneling [17]. In electrodeposition, metal valence electrons also tunnel to dehydrated metal ions, leading to neutralization. The physical reason of electron tunneling is that the wave function of valence electrons in metals does not drop to zero abruptly at the surface but extend above the surface with an exponentially decaying tail [18]. When the wave function tail of the valence electron overlaps with the orbital wave function of hydrated metal ions, the valence electron can tunnel to unoccupied orbital of hydrated metal ions. As a first approximation, the tunneling probability is mainly determined by the overlap of the wave functions. The larger the overlap between the orbital wave function and the valence electron wave function is, the larger the tunneling probability is. The overlap depends very sensitively on the extension of decaying tail and the distance of hydrated metal ions from surface.

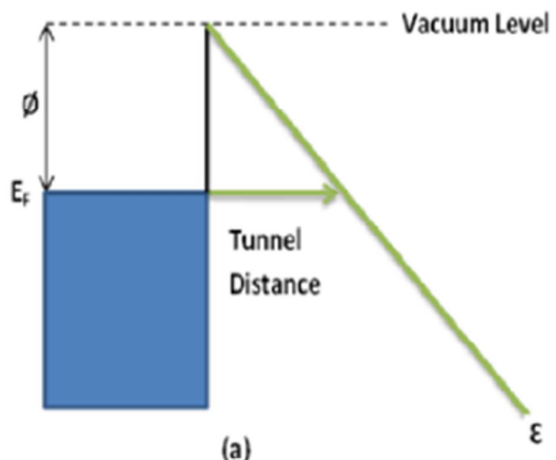

The wavefunction of electrons above the surface decays exponentially according to [19]

$$
\psi \propto \mathrm{e}^{-0.51 \sqrt{\varphi} \cdot \mathrm{d}}
$$

Where $d$ is the distance from metal surface in $\AA$ and $\Phi$ the workfunction in $\mathrm{eV}$. Since the work function of polycrystalline $\mathrm{Fe}(4.67 \mathrm{eV})$ is smaller than that of polycrystalline $\mathrm{Co}(5.0 \mathrm{eV})$ and polycrystalline $\mathrm{Ni}(5.15 \mathrm{eV})$ [20] the valence electron wavefunction of Fe decays less abruptly and extends more above the surface than that of $\mathrm{Co}$ and Ni. The structure of hydrated $\mathrm{Co}$ and $\mathrm{Ni}$ ions is octahedron [21]. When hydrated $\mathrm{Co}$ and $\mathrm{Ni}$ ions approach to and arrive at metal surface, the structure of hydrated metal ions is distorted by electron gas at metal surface. In this case, the distance of hydrated Co and $\mathrm{Ni}$ ions from surface should be same. Therefore the wave function overlap depends mainly on the extension of metal wave function. Because the wave function of Co extends more above the surface than that of $\mathrm{Ni}$, the overlap of Co metal wave function with the orbital wave function of hydrated Co ions is larger than the overlap of $\mathrm{Ni}$ metal wave function with hydrated $\mathrm{Ni}$ ions. Therefore the probability of Co metal valence electron transferring to hydrated Co ions will be higher than that of $\mathrm{Ni}$ metal valence electron transferring to hydrated $\mathrm{Ni}$ ions. This leads to a higher charge transfer coefficient of Co. As a result, the current density for deposition of $\mathrm{Co}$ is higher than that of $\mathrm{Ni}$ and the current density of Fe is higher than that of Co.
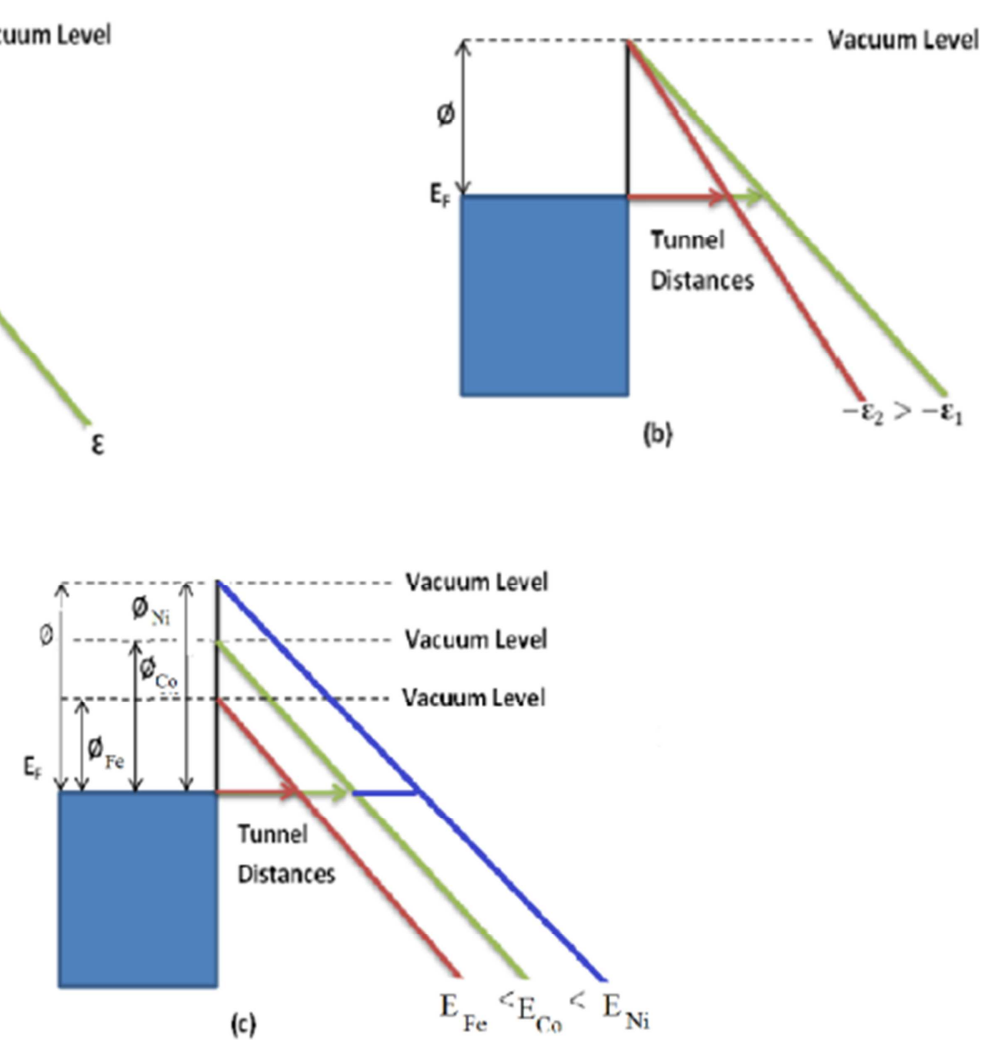

Fig. 5. Schematic diagram of field emission barrier, The height of the potential barrier is determined by the workfunction of the metal ø and slope below the vacuum level shows the applied electric field $\varepsilon$ and the electron tunneling will occur at or near the Fermi level (a), dependence of the barrier width on applied electric field (b), width of potential barrier for different work functions (c) [22]. 
It is well-known that increasing the applied potential increases the current density as shown in the right side of Fig. 5a. This leads to the more rapid change of the potential of electrons outside the metal, as shown in Fig. 5b. In this case, the slope of electron potential with a higher electric field is steeper than that of a small electric field. The barrier width through which electrons tunnel becomes thinner and electron tunneling occurs more easily. Therefore, the current density increases with increasing the applied potential. The work function of metal can have an effect on the barrier width that electrons tunnel through. Fig. 5c shows that under the same applied potential (meaning that there is the same electric field strength in the double layer) the barrier width for the metal with small work function becomes thinner and electron tunneling takes place more easily. Therefore under the same applied potential, there is a higher current density for deposition of nanowires of the metal with a smaller work function. The structure of hydrated metal ions from surface can also be responsible for growth rate of metal nanowires. Different deposition technique like cyclic voltammetry will also be used in our further study about growth mechanism in near future.

\section{Conclusions}

The current density of depositing Fe nanowires is higher than that of depositing $\mathrm{Co}$ as well as $\mathrm{Ni}$ nanowires under the same overpotentials, $\mathrm{pH}$, concentration of metal ions and temperature. This can be attributed to the smaller work function of Fe metal, compared to that of $\mathrm{Co}$ and $\mathrm{Ni}$. For the surface with a smaller work function, the wave function of valence electron extends more above the surface. This causes a larger probability of electron transferring from Fe surface to hydrated $\mathrm{Fe}$ ions, leading to a higher charge transfer coefficient and so a higher current density.

\section{References}

[1] G. Riveros, S. Green, A. Cortes, H. Gómez, R. E. Marotti, E. A. Dalchiele, Silver nanowire arrays electrochemically grown into nanoporous anodic alumina templates, Nanotechnology, 17 (2006) 561 .

[2] S. Valizadeh, J. M. George, P. Leisner, L. Hultman, Electrochemical synthesis of $\mathrm{Ag} / \mathrm{Co}$ multilayered nanowires in porous polycarbonate membranes, Thin Solid Films, 402 (2002) 262-271.

[3] S. B. Chaney, S. Shanmukh, R. A. Dluhy, Y.-P. Zhao, Aligned silver nanorod arrays produce high sensitivity surface-enhanced Raman spectroscopy substrates, Applied Physics Letters, 87 (2005) -

[4] C. R. Martin, Nanomaterials: A Membrane-Based Synthetic Approach, Science, 266 (1994) 1961-1966.
[5] Han G C, Zong B Y, W. Y. H, IEEE Trans. Magn, (2002) 25622564.

[6] M. Z. D. J. Sellmyer, R. Skomski, Magnetism of Fe, Co, and Ni Nanowires in Self-Assembled Arrays, J. Phys. Condens. Matter, 13 (2001) R433-R460

[7] A. Huczko, Template-based synthesis of nanomaterials, Applied Physics A, 70 (2000) 365-376.

[8] M. Tian, J. Wang, J. Kurtz, T. E. Mallouk, M. H. W. Chan, Electrochemical Growth of Single-Crystal Metal Nanowires via a Two-Dimensional Nucleation and Growth Mechanism, Nano Letters, 3 (2003) 919.

[9] M. Tan, X. Chen, Growth Mechanism of Single Crystal Nanowires of fcc Metals ( $\mathrm{Ag}, \mathrm{Cu}, \mathrm{Ni})$ and hep Metal $(\mathrm{Co})$ Electrodeposited, Journal of The Electrochemical Society, 159 (2012) K15.

[10] S. K. Lower, Electrochemistry, Simon Fraser University, Textbook.

[11] H. Masuda, K. Fukuda, Ordered Metal Nanohole Arrays Made by a Two-Step Replication of Honeycomb Structures of Anodic Alumina, Science, 268 (1995) 1466.

[12] T. Mehmood, B. Shahzad Khan, A. Mukhtar, X. Chen, P. Yi, M. Tan, Mechanism for formation of fcc-cobalt nanowires in electrodeposition at ambient temperature, Materials Letters, 130 (2014) 256-258.

[13] A. Mukhtar, T. Mehmood, B. S. Khan, M. Tan, Effect of Co2+ concentration on the crystal structure of electrodeposited Co nanowires, Journal of Crystal Growth, 441 (2016) 26-32.

[14] M. Paunovic, M. Schlesinger, Fundamentals of Electrochemical Deposition, Fundamentals of Electrochemical Deposition, New York: Wiley., (1998).

[15] M. Paunovic, M. Schlesinger, Fundamentals of Electrochemical Deposition, Wiley, New York, 1998.

[16] H. Kita, Periodic Variation of Exchange Current Density of Hydrogen Electrode Reaction with Atomic Number and Reaction Mechanism, Journal of the electrochmical Society, 113 (1966) 1096.

[17] J. C. Tully, N. H. Tolk, in: J. C. T. N. H. Tolk, W. Heiland, C. W White (Ed.) Inelastic Particle-Surface Collision, Academic Press INC, New York, 1977, pp. 105.

[18] L. E. C. v. d. Leemput, H. v. Kempen, Scanning tunnelling microscopy, Rep. Prog. Phys., 55 (1992) 1165.

[19] S. J. Garrett, Introduction to Surface Analysis, the Michigan State University, Chemistry Department, East Lansing.

[20] J. Speight, Lange's Handbook of Chemistry, 16th ed ed., McGraw-Hill Professional, Boston, MA, 2004.

[21] I. Persson, Hydrated metal ions in aqueous solution: How regular are their structures? Pure Appl. Chem., 82 (2010) 1901.

[22] B. Shahzad Khan, T. Mehmood, A. Mukhtar, M. Tan, Effect of workfunction on the growth of electrodeposited $\mathrm{Cu}, \mathrm{Ni}$ and $\mathrm{Co}$ nanowires, Materials Letters, 137 (2014) 13-16. 